\title{
Full-thickness skin graft vs. synthetic mesh in the repair of giant incisional hernia: a randomized controlled multicenter study
}

\author{
L. Clay ${ }^{1} \cdot$ B. Stark ${ }^{2} \cdot$ U. Gunnarsson ${ }^{3} \cdot$ K. Strigård ${ }^{3}$
}

Received: 15 February 2017 / Accepted: 9 December 2017 / Published online: 15 December 2017

(c) The Author(s) 2017. This article is an open access publication

\begin{abstract}
Purpose Repair of large incisional hernias includes the implantation of a synthetic mesh, but this may lead to pain, stiffness, infection and enterocutaneous fistulae. Autologous full-thickness skin graft as on-lay reinforcement has been tested in eight high-risk patients in a proof-of-concept study, with satisfactory results. In this multicenter randomized study, the use of skin graft was compared to synthetic mesh in giant ventral hernia repair.

Methods Non-smoking patients with a ventral hernia $>10 \mathrm{~cm}$ wide were randomized to repair using an on-lay autologous full-thickness skin graft or a synthetic mesh. The primary endpoint was surgical site complications during the first 3 months. A secondary endpoint was patient comfort. Fifty-three patients were included. Clinical evaluation was performed at a 3-month follow-up appointment.

Results There were fewer patients in the skin graft group reporting discomfort: $3(13 \%)$ vs. $12(43 \%)(p=0.016)$. Skin graft patients had less pain and a better general improvement. No difference was seen regarding seroma; 13 (54\%) vs. 13 (46\%), or subcutaneous wound infection; $5(20 \%)$ vs. 7 (25\%). One recurrence appeared in each group. Three patients in the skin graft group and two in the synthetic mesh group were admitted to the intensive care unit.

Conclusion No difference was seen for the primary endpoint short-term surgical complication. Full-thickness skin graft appears to be a reliable material for ventral hernia repair producing no more complications than when using synthetic mesh. Patients repaired with a skin graft have less subjective abdominal wall symptoms.
\end{abstract}

Keywords Incisional hernia Ventral hernia $\cdot$ Full-thickness skin · Abdominal wall reconstruction · Hernia complication · Infection

\section{Introduction}

The risk for developing an incisional hernia following elective abdominal surgery is approximately $10 \%$ [1]. This risk increases up to $33 \%$ following acute abdominal surgery [2]. Individuals who smoke, or suffer from obesity or diabetes have an even higher risk for developing a hernia following abdominal surgery $[3,4]$. Those who develop a giant ventral

\section{K. Strigård \\ karin.strigard@umu.se \\ 1 Department of Clinical Science, Intervention and Technology (CLINTEC), H9, Karolinska Institutet, 17164 Stockholm, Sweden}

2 Department of Molecular Medicine and Surgery, Karolinska Institutet, 17164 Stockholm, Sweden

3 Department of Surgical and Perioperative Sciences, Umeå University, 90785 Umeå, Sweden hernia often experience a lower quality of life not only due to the hernia's appearance, but also because of pain, discomfort, hygiene difficulties and inability to perform daily activities [5]. The most serious complication is incarceration of abdominal contents with impaired blood supply and subsequent intestinal necrosis.

The current standard method used for the repair of a large incisional hernia involves implantation of a synthetic mesh to reinforce the abdominal wall [6]. However, giant hernias cannot be successfully repaired using standard methods due to their complexity [6], and they often follow previous unsuccessful repair. There is no common definition of giant hernia. In this study, as in several others, a giant ventral hernia was defined as one greater than $10 \mathrm{~cm}$ wide. The use of mesh in standard hernia repair reduces the risk for recurrence to approximately $10 \%$, but for giant hernias the recurrence rate ranges between 10 and 30\% [7]. Synthetic mesh may also result in chronic pain, infection 
and enterocutaneous fistula formation. Impairment of abdominal wall function with decreased muscle strength and flexibility [8] is another common consequence of giant ventral hernia. Full-thickness skin graft as an on-lay prosthesis has been evaluated in a prospective feasibility study in a limited cohort [9] of complex high-risk cases, with favorable results and no obvious side-effects. These patients were unsuitable for application of foreign material due to immune-depression and/or diseases with altered immune-response, such as Crohn's disease. One patient from this study has been re-operated for reasons other than hernia several years later, and at that time a sample from the previously transplanted skin mesh was taken for histology. Examination revealed fibrous tissue only with no skin adnexes. After a median follow-up of 33 months, no further active follow-up has been made. No long-term complications have come to the attention of the authors. One patient died a few years after surgery due to heart disease. An alternative experimental method for the reconstruction of complex abdominal wall defects is the initial coverage of exposed intestines with a skin graft and subsequent closure of the fascia by invagination of the tube [10]. Over the last century, skin grafts have occasionally been used in reinforcement of the abdominal wall [11]. Early studies from the 1940s have reported the use of full-thickness skin graft for inguinal hernia [12]. The shoelace method was first described in the 1950s [13] and is still used at some centers. The use of a full-thickness skin graft with multiple 5-10 $\mathrm{mm}$ scalpel incisions in mesh formation, as an on-lay implant for reinforcement, not just to cover the open defect, has not previously been described in humans.

Giant ventral hernia is a complex condition and wound complications may be expected to occur after $40-50 \%$ of repair procedures [14]. It is not known if this high frequency is caused by habitual factors, complexity of the procedure, or a consequence of the synthetic material implanted. Biologic material is used in some centers, but the cost of this material is very high, easily exceeding 10,000 Euros for each patient. The use of autologous skin grafting may be an economical way to avoid complications related to synthetic material.

In this multicenter prospective randomized study, an autologous full-thickness skin graft was used as an on-lay prosthesis and compared with conventional abdominal wall reinforcement with synthetic mesh in giant ventral hernia repair. The primary endpoint was surgical site complication, i.e., bleeding, infection, seroma and sinuses, during the first three postoperative months. A secondary endpoint was patient comfort. Our hypothesis was that the use of an autologous full-thickness skin graft in giant ventral hernia repair carries a lower risk for surgical complications than synthetic mesh.

\section{Methods}

\section{Study design}

The study was designed as a randomized controlled multicenter study carried out at two university departments serving as tertiary referral centers for advanced reconstructive surgery of the abdominal wall. Follow-up was performed by a senior surgeon not involved in the study and blinded to the surgical procedure used. Inclusion criteria were: hernia measuring at least $10 \mathrm{~cm}$ across (clinically or measured on a CT scan); 18 years of age or older; and non-smoking for at least 3 months prior to surgery. Smokers were excluded due to the negative side-effects seen for wound and anastomotic healing $[15,16]$. In some cases, patient-related factors, such as obesity made hernia size difficult to assess by clinical measurement. All patients underwent a CT scan. Patients not eligible for the study or not wishing to be included were operated with synthetic mesh as is routine practice. There were only a few patients who opted not to participate in the study due to unwillingness to adhere to the follow-up protocol. Inclusion began December 2009 and ended August 2013. Patients were randomized to either repair with a synthetic mesh in the best possible position or repair with a full-thickness skin graft in an on-lay position. When using synthetic mesh, the sub-lay repair technique was used whenever possible, while on-lay repair was used when the retro-muscular space was densely obliterated from previous surgery or along with stoma reversal where the mesh was situated as far from the intestine as possible.

An experienced research nurse administered the randomization process. Initially, 50 envelopes, each containing a paper printed with either "skin" or "mesh" (25 of each) were prepared. On the day prior to surgery, the research nurse randomly selected and opened one of the unmarked envelopes revealing the randomized surgical method to be allocated to the patient in question, and thereafter informed the surgical team. When obtaining written informed consent, all participants agreed to forgo knowledge of which surgical method was to be used in their repair until completion of the study. All patients had had a previous midline incision.

This study was approved by the Regional Board of Ethics (D.nr. 2009/227-31/3) at the Karolinska Institutet, March 11,2009 . The study was registered at ClinicalTrials.gov (ID NCT01413412) August, 9, 2011. The ethical standards followed the principles of the Declaration of Helsinki.

\section{Preoperative procedures}

All patients were individually fitted with an elastic girdle from Nordic Care $\odot$. This girdle has a wide velcro 
attachment surface for comfort and support. The elastic girdle was worn $24 \mathrm{~h}$ a day for 3 months prior to surgery. All patients underwent CT scanning of the abdominal wall. The following were registered: gender; age at time of surgery; weight; body mass index (BMI); blood pressure and pulse. Patients underwent a clinical examination to ascertain the palpable dimensions of their hernia. At the same time the quality of skin was assessed for potential skin transplant. Size of the hernia was measured with tape with the patient in the supine position. Most patients underwent pulmonary function tests and many of them cardiac evaluation with perfusion analysis. On the morning of surgery, all patients were given trimethoprim-sulphonamide $800 \mathrm{mg}$ and metronidazole $1200 \mathrm{mg}$ orally. Patients randomized to a full-thickness skin graft also received clindamycin $300 \mathrm{mg}$ orally. Prior to intubation, all patients received an epidural catheter for per- and postoperative analgesia.

\section{Intraoperative procedures}

All procedures were performed by one of two surgeons experienced in hernia and colorectal surgery as the main surgeon. These two surgeons had similar experience of both conventional and full-thickness skin graft methods. In most cases where a full-thickness skin graft was used, a plastic surgeon experienced in hernia surgery also participated. Following induction and intubation, the patients were examined with a tape measure to obtain clinical measurements of the hernia in the relaxed state. Peak airway pressure was noted prior to the start of surgery, as well as before and after reconstruction of the abdominal wall, and this was used as a proxy for tension during closure of the hernia. The skin wound was sealed in three layers with absorbable monofilament sutures. Placement of the synthetic mesh was at the discretion of the main surgeon, taking anatomical circumstances and any previously implanted mesh into consideration.

\section{Reconstruction with a full-thickness skin graft}

An elliptical patch was marked out on the skin overlying the hernia defect and encompassing the previous abdominal incisional scar (Fig. 1). Once the autologous skin patch had been excised, all subcutaneous fat was removed and the full-thickness skin graft fenestrated meshwise with 5-10 mm incisions using a scalpel. The goal was to obtain a patch overlapping the defect by $5 \mathrm{~cm}$ in every direction. In some cases only $3 \mathrm{~cm}$ overlap was achieved. If the midline of the proposed graft had poor skin quality, a paramedial graft was formed using skin up to the midline. When a stoma was concurrently closed at the time of hernia repair, skin bordering the stoma area was used. In no case was there difficulty in skin closure after excision of the graft. Pending

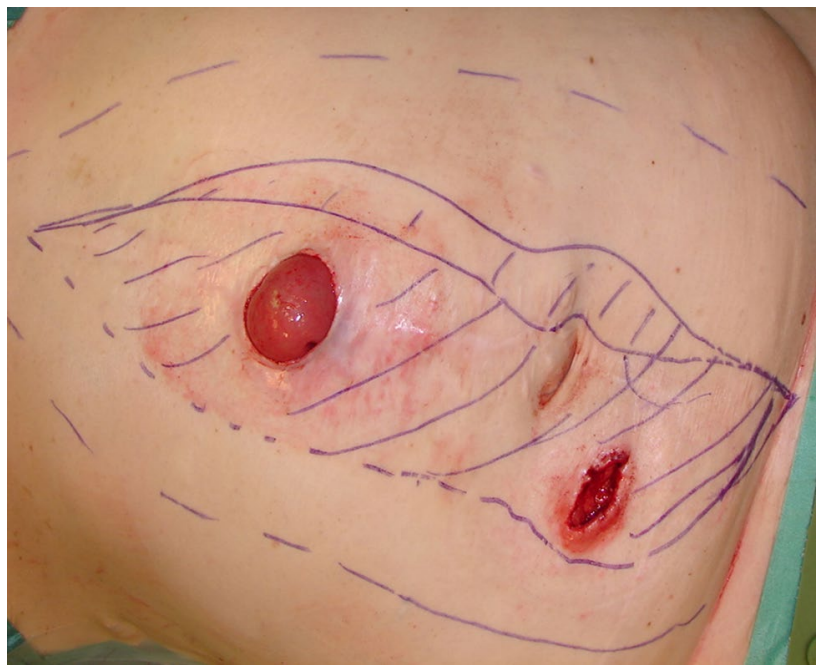

Fig. 1 Giant ventral hernia with the defect marked (outer line) as well as the proposed skin graft (inner line)

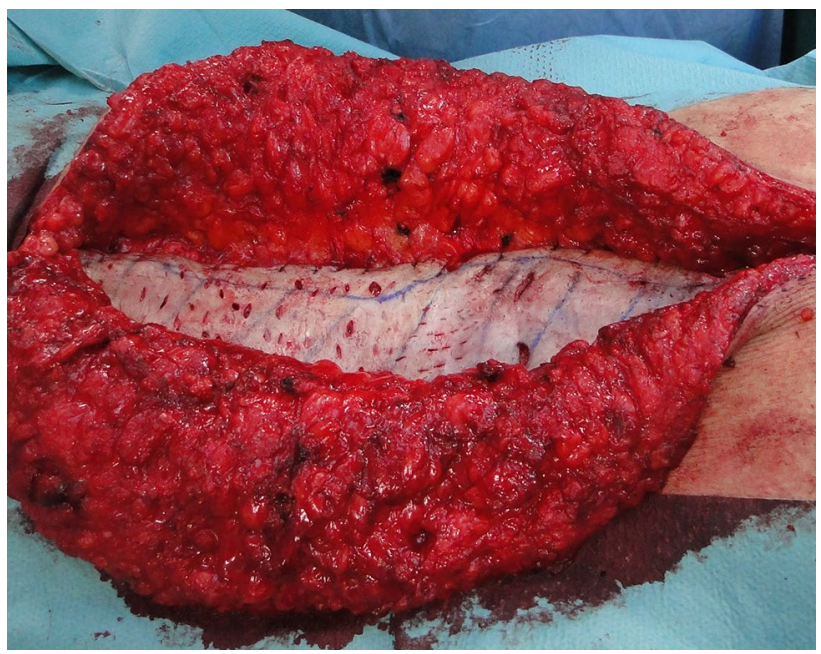

Fig. 2 The full-thickness skin graft in place as an on-lay prosthesis

implantation, the full-thickness skin graft was rolled up in surgical gauze soaked in a solution of equal parts physiological saline and hydrogen peroxide.

The hernia defect was then closed using slowly absorbable 0.0 monofilament suture (PDS). The continuous closure suture was knotted after every sixth stitch (8). In the event of undue tension in the abdominal wall, releasing incisions were performed according to Chevrel [17]. The full-thickness skin graft was then stretched over the sutured defect so as to obtain a $5 \mathrm{~cm}$ overlap around the defect margin (Fig. 2). The graft was sutured to the underlying abdominal fascial wall under tension using individual stitches of absorbable 4.0 monofilament suture. The space beneath was flushed with hydrogen peroxide using a venous cannula. Two 
exudrains were inserted, since this was used in the proof-ofconcept study [9].

\section{Reconstruction with synthetic mesh}

Whenever possible, the retromuscular space was used according to Rives-Stoppa [18]. A lightweight polypropylene mesh was placed overlapping the hernia defect margin by at least $5 \mathrm{~cm}$ in every direction. No sutures were used to secure the mesh as it was considered to be immobilized by the abdominal pressure exerted on the space by the rectus muscles. The hernia defect was closed with 2-0 absorbable monofilament suture. The fascial defect was then closed with non-absorbable 0.0 monofilament suture. To gain a closure without tension, releasing incisions in the external fascia according to Chevrel were used [19].

In cases where the retromuscular space was obliterated or otherwise too risky or difficult to safely access without undue risk for complication, the rectus fascia was closed with non-absorbable 0.0 monofilament suture. The repair was then reinforced as an "on-lay" according to Chevrel using heavyweight polypropylene mesh trimmed to size and placed on the rectus muscle aponeurosis [19]. Even in these cases, releasing incisions in the external fascia were used. The mesh was fixed in place with absorbable 2-0 monofilament suture in double rows $1-2 \mathrm{~cm}$ from the midline closure.

Excess skin was removed to achieve an exact closure. No drains were used according to present routine in the hospitals participating.

\section{Postoperative management}

The specially fitted elastic abdominal girdle was reapplied prior to extubation and worn $24 \mathrm{~h}$ a day for 6 weeks, followed by another 6 weeks during the day only. Mobilization was encouraged the day after surgery. Patients in the skin group were treated postoperatively with oral clindamycin $300 \mathrm{mg}, 3$ times a day for 10 days, and both groups were given $5000 \mathrm{IE}$ low molecular weight heparin (LMWH) subcutaneously for 7 days. Nurses on the ward were not informed as to why some patients had drains or why only some received postoperative antibiotics. The reason for the incongruence between the various surgical alternatives was a result of our initial experience using full-thickness skin grafts [9].

All patients were scheduled for follow-up with a clinical evaluation 3 months after surgery. The surgeons who examined the patients were blinded to the surgical technique used. Patients were examined regarding their vital signs, weight, abdominal wall integrity, appearance of the surgical result (wound healing, excess of skin, symmetry of the abdominal wall and if there was any sign of recurrence), and for the presence of discomfort or pain. The presence of seroma, fistula, infection, other surgical complications as well as discomfort in the abdominal wall, were answered by a "yes" or "no". If "yes", a comment was mandatory. Patients were also asked about their subjective experience of improvement using a VAS scale graded from 0 to 10 where 10 was the best possible outcome. A VAS scale was also used to evaluate the experience of pain from the abdominal wall where 0 was no pain and 10 maximal pain. In cases of recurrence, the hernia dimensions were noted.

\section{Outcome}

The primary outcome was surgical site complication during the first 3 months, including early recurrence, seroma, subcutaneous fistulae, infection and bleeding. A secondary outcome was patient comfort.

\section{Statistical analysis}

The intended sample size was based on the assumption that surgical site complications would be less in the group operated without synthetic material. The complication rate was estimated at $50 \%$ in the synthetic mesh group and $20 \%$ in the full-thickness skin graft group at the 3-month follow-up visit. Fifty patients were required for $80 \%$ power and $95 \%$ significance.

The trial was registered at ClinicalTrials.gov (ID NCT01413412).

Statistics were analyzed using Statistica ${ }^{\circledR}$ version 12 (Statsoft, Tulsa, USA). Non-parametric statistics were generally used. The hernia area was calculated as $\pi \times$ hernia length $/ 2 \times$ hernia width/2. Differences in proportions were calculated using the Chi-square test. Subjective improvement, measured by a VAS scale, was calculated by the Mann-Whitney $U$ test.

\section{Results}

One patient was withdrawn after randomization due to increasing comorbidity making her unfit for anesthesia. She was compensated for by 3 new randomized patients, leaving 52 patients for evaluation. Ten more envelopes were prepared after the first 50 randomizations to allow for the patient that was withdrawn. All three new patients were by chance randomized to the synthetic mesh group. There was thus, a slight imbalance with 24 patients in the full-thickness skin graft group and 28 in the synthetic mesh group. Inclusions and exclusions are shown in the CONSORT diagram in Fig. 3.

Basic patient characteristics and BMI were similar in the two groups (Table 1). There was one patient with stoma in each group and stoma reversal was performed in two cases 


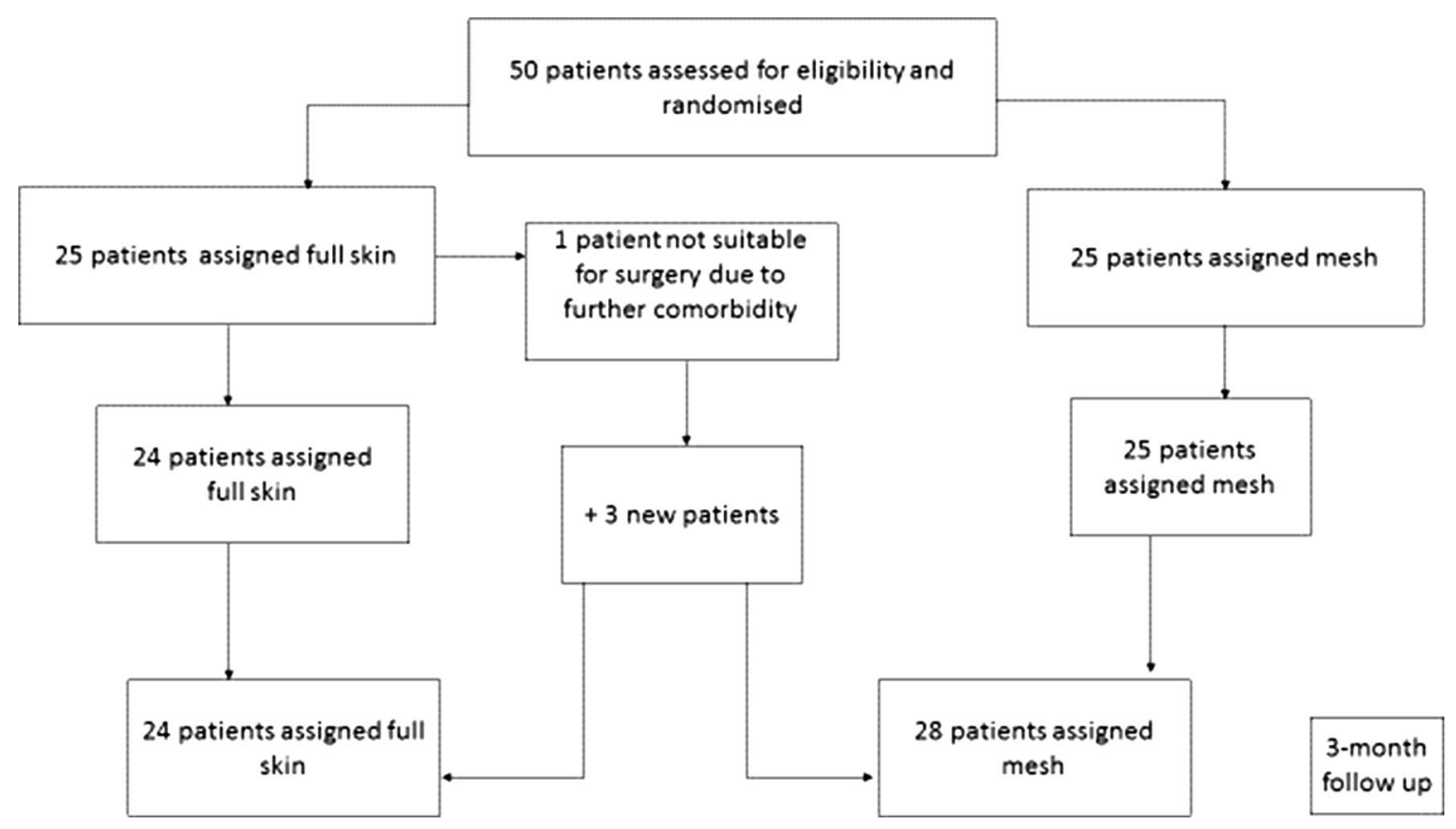

Fig. 3 CONSORT diagram of the study

Table 1 Basic data

\begin{tabular}{llll}
\hline & Full-thickness skin & Mesh & $p$ \\
\hline BMI & $31.8(5.2)$ & $31.4(6.2)$ & 0.75 \\
Age & $62.9(9.3)$ & $62.3(9.1)$ & 0.81 \\
Gender (male/female) & $12 / 12$ & $15 / 13$ & 0.80 \\
Width of hernia & $14(4.8)$ & $13.7(6.5)$ & 0.70 \\
Area of hernia & $181.4(100.1)$ & $153.2(89.6)$ & 0.39 \\
\hline
\end{tabular}

Data mean (SD). For gender, the actual number of males and females are shown. The width of the hernia is in $\mathrm{cm}$ and area in $\mathrm{cm}^{2}$

in the skin graft group and one case in the mesh group. The transverse diameter and area of the hernia are also shown in Table 1 . The area was slightly larger in the full-thickness skin graft group. At evaluation, only on-lay (8 patients) and sub-lay (20 patients) mesh placements were used in this study.

Preoperative CT scans did not reveal any unknown concomitant intra-abdominal disease.

At surgery a lightweight mesh was used in sub-lay position in 20 cases. When the on-lay procedure was performed, a heavyweight mesh was used (8 cases). The median duration of surgery was 186 min (range 130-262) for on-lay reconstruction, $178 \mathrm{~min}$ (range 108-324) for sub-lay and 224 min (range 128-414) for full-thickness skin graft.

The drains in the full-thickness skin graft group were removed after 2-5 days. No patient went home with a drain. Drains were removed when the fluid loss was less than $50 \mathrm{ml}$ each day or at the latest Day 5 postoperatively.
To the question "Do you experience any discomfort at the surgical site?", 3 patients answered "yes" in the fullthickness skin graft group and 12 "yes" in the mesh group $(p=0.016)$. Patients in the skin graft group reported less pain compared to the mesh group $3(13 \%)$ vs. 12 (43\%). Patients operated with full-thickness skin grafts rated a higher degree of subjective improvement in their abdominal wall function compared to those operated with a synthetic mesh (median 8 respective $7 ; p=0.038$ ). There was one hernia recurrence in each group. No difference was seen for surgical site complications overall (skin graft 16/24 vs. mesh 17/28). Two patients in the mesh group were reoperated due to bleeding. No patient in the full-thickness skin graft group was reoperated. There was no difference seen regarding the occurrence of clinical seroma: $13(54 \%)$ in the skin group and $13(46 \%)$ in the mesh group $(p=0.58)$. Superficial sinuses, defined as small non-healed wounds with non-infected secretion, were equally common in the two treatment groups $(p=0.46)$. There was no difference in subcutaneous wound infection with $5(20 \%)$ patients in the skin graft group and $7(25 \%)$ in the mesh group $(p=0.72)$ (Table 2). All but one infection were superficial or limited to the subcutaneous tissue. The patient with a deeper infection had received a full-thickness skin graft. It was not necessary to remove the skin graft, but the overlying skin wound was reconstructed using a cutaneous flap. One of the patients in the mesh repair group had a subcutaneous infection that required surgical revision under general anesthesia. All the patients classified as suffering from infection were treated with clindamycin. Random samples for bacterial culture 
Table 2 Outcome at 3-month follow-up

\begin{tabular}{lccc}
\hline Outcome & $\begin{array}{l}\text { Full-thickness } \\
\text { skin graft, } \\
n=24\end{array}$ & $\begin{array}{l}\text { Synthetic } \\
\text { mesh, } \\
n=28\end{array}$ & $p$ \\
\hline $\begin{array}{l}\text { Discomfort at surgical site } \\
\quad \text { yes/no) }\end{array}$ & 3 & 12 & 0.016 \\
$\begin{array}{l}\text { Subjective improvement } \\
\quad \text { median) }\end{array}$ & 8 & 7 & 0.038 \\
Pain at surgical site & 3 & 12 & 0.016 \\
Hernia recurrence & 1 & 1 & 0.91 \\
Surgical site complications & 16 & 17 & 0.66 \\
Seroma & 13 & 13 & 0.58 \\
Superficial sinuses & 2 & 1 & 0.46 \\
Subcutaneous wound infection & 5 & 7 & 0.72 \\
Reoperation due to bleeding & 0 & 2 & - \\
\hline
\end{tabular}

The number of surgical site complications at 3-month follow-up in the full-thickness skin graft versus the synthetic mesh group. $p$ values are calculated by the $\chi^{2}$ test with exception for "Subjective improvement" which shows the median and $p$ value calculated by the MannWhitney $U$ test

were taken from the skin graft at surgery. In one case there was sparse growth of normal skin flora, otherwise there was no growth in any of the other samples.

The difference in airway pressure with muscle relaxation before and after surgical reconstruction of the abdominal wall ranged between -3 and $+9 \mathrm{cmH}_{2} \mathrm{O}$ with a median of $+3 \mathrm{cmH}_{2} \mathrm{O}$. The two highest differences of +9 and +7 $\mathrm{cmH}_{2} \mathrm{O}$ were seen in the full-thickness skin transplant group. The initial airway pressure ranged between +11 and +27 $\mathrm{cmH}_{2} \mathrm{O}$.

There were three patients in the full-thickness skin graft group and two in the synthetic mesh group who were admitted to the intensive care unit (ICU). Of the patients in the full-thickness skin group one required ICU care for aspiration (22 days in the ICU), one suffered a myocardial infarction (28 days in the ICU), and one was due to respiratory failure ( 9 days in the ICU). In the mesh group, there were two with respiratory failure ( 3 and 8 days in the ICU).

There was one recurrence in each group.

\section{Discussion}

The primary endpoint of this study, surgical complication, did not differ between repair with full-thickness skin graft and repair with synthetic mesh. Accordingly, the hypothesis that autologous material would be favorable regarding tissue remodeling and repair with fewer infections was therefore not statistically supported, even if there were numerically somewhat fewer wound infections in the full-thickness skin transplant group. Patients allocated to the mesh group experienced more discomfort and pain at the 3-month follow-up visit and rated a lower degree of improvement in their abdominal wall function, as rated on a VAS scale. This experience of discomfort was defined as a feeling from the abdominal wall and was not related to any surgical site complication. Reasons for this could be better integration of autologous material or less pronounced inflammation. When there is an uneventful postoperative course after synthetic mesh implantation to reinforce the abdominal wall, ingrowth of tissue into the mesh involves remodeling of connective tissue structures. Consequently, this can lead to a rather rigid plate that does not follow abdominal wall flexion. Most of the mesh implants were placed in a sub-lay position in the preperitoneal space, but these patients still had symptoms of discomfort. Reinforcement using both on-lay and sub-lay techniques may be a weakness of the study design, but we chose to do this in order to offer patients the best possible repair in both arms. An alternative would have been to place all implants in the on-lay position. In a previous study, however, abdominal muscle strength was independent of whether the mesh was placed in the on-lay, sub-lay or IPOM positions [19], but seroma was more common with on-lay repair.

As in other studies [20], seroma occurred frequently with no difference between the groups. In this study, we actively looked for seromas and registered also events that did not need any intervention, which was the majority. The use of drains did not seem to prevent or promote seroma formation. Full-thickness skin graft patients all received two exudrains during surgery, whereas patients allocated to the mesh arm did not receive a drain. No conclusions can be drawn from this difference since there were too few patients in each group and no randomization was made for this treatment step. Since a few patients had concurrent stoma closure, some surgeons today would argue that biologic prosthetic material should have been chosen [21]. This study does not support that opinion because the frequency of surgical complications did not differ between the two groups. One could argue that commercial biologic material is sterile, whereas the patient's own skin is not. However, random cultured samples from implanted skin grafts taken immediately before implantation showed no bacterial growth or very low titers of skin bacteria. There were no intra-abdominal infections observed in this study. However, one subcutaneous infection necessitated surgical revision. Since both synthetic mesh and autologous skin graft performed equally, the high cost of biologic implants is clearly not justified.

After 3 months, there was one recurrence in each group, which is to be expected in a cohort of patients undergoing repair for giant ventral hernia [7]. One recurrence involved a patient who later admitted to smoking both pre- and postoperatively, but denied this when being assessed prior to surgery. While patients were examined for the presence of recurrence, it was not a primary endpoint in this 3-month short-term follow-up. The recurrence rate would probably 
be low in both surgical arms, since there was only one recurrence in the proof-of-concept study [9]. A study with recurrence as the main end point would need to be extremely large. Impaired tissue remodeling and repair is well documented among smokers $[3,22]$ and was thus an exclusion criterion in this study.

The technique used for closing the hernia in this study involved a degree of tension. Despite performing small releasing incisions (ad modum Chevrel) in the external fascia, the abdominal wall was still closed with some tension. The relative increase in airway pressure, measured by the ventilator, was used as a proxy for tension when closing the abdominal wall. Despite tension, there were only four patients requiring ICU care because of compromised breathing, one of which was caused by aspiration. The proportion of patients in this study needing postoperative ICU care after giant ventral hernia repair was low, and probably reflects our very close cooperation with the anesthetist. Care was taken to avoid excessive fluid administration, and active breathing exercises were initiated as soon as possible upon awakening. None of the cases required component separation, which may indicate that a degree of residual tension is not as harmful as suggested in some recent publications [23, 24]. It is also important to note that component separation per se carries with it a morbidity risk, not least poor wound healing.

The hypothesis that wound healing would be improved by the use of a full-thickness skin graft was not confirmed. Even so, severe complications and the need for intensive care due to respiratory complications were less frequent compared to other reports $[7,25,26]$. Our study hypothesis was thus rejected. Although the groups showed some differences in the panorama of complications, the expected decrease in wound healing disturbances was not shown, while the frequency of seroma was even higher in the skin graft group compared to the mesh group. One possible explanation for this is that the magnitude of complications may be a consequence of the surgical trauma rather than being associated with the nature of the material used. It is also known that the on-lay technique may be associated to more complications than the sub-lay and IPOM techniques when meshes are used. If the magnitude of surgery is the truth, efforts should be focused on optimizing perfusion and oxygenation and on the mechanisms of formation of seroma. In spite of this, autologous skin grafts still have potential advantages in terms of elasticity, fewer graft infections, and obvious economic superiority.

A limiting factor with the use of the full-thickness skingrafting technique is the availability of skin with good quality. In giant ventral hernia patients, there is usually enough excess skin as a result of the giant hernia itself to allow for excision of a sufficiently sized skin graft. In cases where the skin is damaged, or of poor quality, it is possible to harvest full-thickness skin grafts from the leg or upper arm.
This has been done in some procedures not included in this study. The possibility of using skin grafts in other locations in hernia surgery is now the subject of research. Skin grafts have been used in animal models since the beginning of the twentieth century [27]. There are also experimental studies investigating skin grafts used with a shoelace technique [28]. In these studies, the hypothesis is that the surrounding tissue will infiltrate the tiny skin grafts. The procedure is consequently performed with high tension for maximal ingrowth in contrast to this study where full-thickness skin grafts were conceptually used as ordinary reinforcement material.

In conclusion, autologous full-thickness skin grafts are reliable as material for strengthening the abdominal wall in ventral hernia repair, with similar outcomes and complications compared to using synthetic mesh. Patients operated with a skin graft also experience a more comfortable recovery following surgery. The use of autologous material is cheaper than synthetic mesh, and much cheaper than commercial biologic materials. Furthermore, skin grafting is probably preferable from a histo-immunologic perspective, since it involves the implantation of the patient's own tissue. The value of skin grafting as regards hernia recurrence in the long term has yet to be determined at long-term follow-up.

Acknowledgements We would like to thank our research nurse Mrs. Margareta Michanek for valuable practical help throughout the study.

\section{Compliance with ethical standards}

Funding The study was funded by Stockholm County Council.

Conflict of interest LC, BS, UG and KS declare that they have no conflict of interest.

Human and animal rights All procedures performed in the study involving human participants were in accordance with the ethical standards of the institutional research committee and with the 1964 Helsinki Declaration and its later amendments, or comparable ethical standards.

Ethical approval This study was approved by the Regional Board of Ethics (D.nr. 2009/227-31/3) at the Karolinska Institute, Stockholm, March 11, 2009. The authors confirm that all ongoing and related trials for this intervention are registered. The study was registered at ClinicalTrials.gov (ID NCT01413412). All experiments in the study comply with the current laws of Sweden.

Informed consent Informed consent was obtained from all individual participants included in the study.

Open Access This article is distributed under the terms of the Creative Commons Attribution-NonCommercial 4.0 International License (http://creativecommons.org/licenses/by-nc/4.0/), which permits any noncommercial use, distribution, and reproduction in any medium, provided you give appropriate credit to the original author(s) and the source, provide a link to the Creative Commons license, and indicate if changes were made. 


\section{References}

1. Mudge M, Hughes LE (1985) Incisional hernia: a 10 year prospective study of incidence. Br J Surg 72(1):70-71

2. Argudo N, Pereira JA, Sancho JJ, Membrilla E, Pons MJ, Grande L (2014) Prophylactic synthetic mesh can be safely used to close emergency laparotomies, even in peritonitis. Surgery 156(5):1238-1244

3. Abbas SM, Hill AG (2009) Smoking is a major risk factor for wound dehiscence after midline abdominal incision; case-control study. ANZ J Surg 79(4):247-250

4. Sugerman HJ, Kellum JM Jr, Reines HD, DeMaria EJ, Newsome HH, Lowry JW (1996) Greater risk of incisional hernia with morbidly obese than steroid-dependent patients and low recurrence with prefascial polypropylene mesh. Am J Surg 171(1):80-84

5. Chung L, O'Dwyer PJ (2014) Pain and its effects on physical activity and quality of life before operation in patients undergoing elective inguinal and ventral hernia repair. Am J Surg 208(3):406-411

6. Mathes T, Walgenbach M, Siegel R (2016) Suture versus mesh repair in primary and incisional ventral hernias: a systematic review and meta-analysis. World J Surg 40(4):826-835

7. Bikhchandani J, Fitzgibbons RJ Jr (2013) Repair of giant ventral hernias. Adv Surg 47:1-27

8. Criss CN, Petro CC, Krpata DM, Seafler CM, Lai N, Fiutem J, Novitsky YW, Rosen MJ (2014) Functional abdominal wall reconstruction improves core physiology and quality-of-life. Surgery 156(1):176-182

9. Strigård K, Stark B (2008) Repair of giant abdominal wall hernias with full-thickness skin transplants in high-risk patients. Eur J Plast Surg 31:21-24

10. Stark B, Strigård K (2007) Definitive reconstruction of full-thickness abdominal wall defects initially treated with skin grafting of exposed intestines. Hernia 11(6):533-536

11. Millard DR, Pigott R, Zies P (1996) Free skin grafting of full-thickness defects of abdominal wall. Plast Reconstr Surg 43(6):569-582

12. Mair GB (1946) Analysis of a series of 454 inguinal hernias with special reference to morbidity and recurrence after the whole skingraft method. Br J Surg 34:42-46

13. Gray DB (1951) The fate of buried full thickness skin: an experimental study. Ann Surg 154:205-209

14. Cobb WS, Warren JA, Ewing JA, Burnikel A, Merchant M, Carbonell AM (2015) Open retromuscular mesh repair of complex incisional hernia: predictors of wound events and recurrence. J Am Coll Surg 220(4):606-613

15. Lindström D, Sadr Azodi O, Wladis A, Tønnesen H, Linder S, Nåsell H, Ponzer S, Adami J (2008) Effects of a perioperative smoking cessation intervention on postoperative complications: a randomized trial. Ann Surg. 248(5):739-745

16. Sørensen LT (2012) Wound healing and infection in surgery: the pathophysiological impact of smoking, smoking cessation, and nicotine replacement therapy: a systematic review. Ann Surg 255:1069-1079 (Annals of surgery [0003-4932])

17. Chevrel JP (1979) The treatment of large midline incisional hernias by "overcoat" plasty and prothesis (author's transl). Nouv Presse Med 24(9):695-696

18. Heartsill L, Richards ML, Arfai N, Lee A, Bingener-Casey J, Schwesinger WH, Sirinek KR (2005) Open Rives-Stoppa ventral hernia repair made simple and successful but not for everyone. Hernia 9(2):162-166

19. Johansson H, Gunnarsson U, Strigård K (2011) Different techniques for mesh application give the same abdominal muscle strength. Hernia 15(4):417-421

20. Parameswaran R, Hornby ST, Kingsnorth AN (2013) Medical talc increases the incidence of seroma formation following onlay repair of major abdominal wall hernias. Hernia 17(4):459-463

21. Hadeed JG, Walsh MD, Pappas TN, Pestana IA, Tyler DS, Levinson H, Mantyh C, Jacobs DO, Lagoo-Deenadalayan SA, Erdmann D (2011) Complex abdominal wall hernias: a new classification system and approach to management based on review of 133 consecutive patients. Ann Plast Surg 66(5):497-503

22. Sørensen LT, Hemmingsen UB, Kirkeby LT, Kallehave F, Jørgensen LN (2005) Smoking is a risk factor for incisional hernia. Arch Surg 140(5):119-123

23. Lowe JB 3rd, Lowe JB, Baty JD, Garza JR (2003) Risks associated with "components separation" for closure of complex abdominal wall defects. Plast Reconstr 111(3):1276-1283

24. de Vries Reilingh TS, van Goor H, Rosman C, Bemelmans MH, de Jong D, van Nieuwenhoven EJ, van Engeland MI, Bleichrodt RP (2003) "Components separation technique" for the repair of large abdominal wall hernias. J Am Coll Surg 196(3):32-37

25. Harth KC, Rosen MJ (2010) Endoscopic versus open component separation in complex abdominal wall reconstruction. Am J Surg 199:342-346 (discussion: 346-347)

26. Lowe JB 3rd, Lowe JB, Baty JD, Garza JR (2003) Risks associated with "components separation" for closure of complex abdominal wall defects. Plast Reconstr Surg 111(3):1276-1283 (quiz: 1284-1285; discussion: 1286-1288)

27. Rehn E (1914) Das kutane und subkutane Bindegewebe als plastisches Material. Münch Med Wochenschr 61:118-121

28. Navarrini GC, Russo C, Armaroli R, Guerzoni AP (1987) Skin grafts in the surgical treatment of incisional hernias. It J Surg Sci 17(1):49-53 